\title{
Darcy's law for yield stress fluid flowing through a porous medium
}

\author{
T. Chevalier, C. Chevalier ${ }^{1}$, X. Clain, J.C. Dupla, J. Canou, S. Rodts, and P. \\ Coussot
}

\author{
Université Paris-Est, Laboratoire Navier (UMR 8205), CNRS, ENPC, IFSTTAR, F-77420 Marne- \\ la-Vallée \\ ${ }^{1}$ Université Paris Est, IFSTTAR, Département Géotechnique Eau et Risques, F-77420 Marne-la- \\ Vallée
}

\begin{abstract}
We measured the pressure drop vs flow rate during the flow, in a wide range of velocities, of well controlled yield stress fluids through confined packings of glass beads of different sizes. A detailed analysis of the data makes it possible to extract a general expression for the pressure drop vs flow rate curve through a porous medium as a function of the flow rate and the characteristics of the system. This general law has a form similar to the Herschel-Bulkley model describing the rheological behavior of such fluids in simple shear, i.e. it expresses as the sum of a critical (yielding) pressure drop and a flow rate dependent term. This law involves the rheological parameters of the fluid, one characteristic length of the medium, and two coefficients which only depend on the structure of the porous medium. The first coefficient is related to the path of maximum width throughout the porous medium while the second coefficient reflects the pore size distribution. The values of these coefficients were determined in the case of a granular packing.
\end{abstract}

\section{Introduction}

The flow of non-Newtonian fluids through porous media is of interest for various applications: penetration of glue in the surface porosity of solid materials, injection of muds, slurries or cement grouts to reinforce soils, propagation of blood through kidney and, likely the most economically important application, injection of drilling fluids in rocks either for the reinforcement of the wells or for enhancing oil recovery, etc. Much work has focused on the flow properties of purely viscous non-Newtonian fluids, the simplest example being power-law fluids [1-4]. For example the propagation of a non-Newtonian liquid pushing another one, and the possible instability of the interface leading to fingering, is a subject of importance which has already been studied [5-6]. Another aspect concerns the effect of inertia which can further complicate the analysis [7]. However there is a wide range of injected fluids, and in particular in oil industry, which exhibit a strongly non-Newtonian behavior, i.e. they are yield stress fluids which flow like liquid only when the stress applied to them is larger than a critical value. These fluids are emulsions, foams, clayey suspensions or even some polymeric gels. As far as the flow of such fluids through porous media is concerned this yield stress character is likely to induce specific properties which are so far poorly identified. The basic knowledge of the pressure drop needed to induce a steady inertia-less flow of a yield stress fluid through a porous medium is so far not very well developed. In this paper we mainly focus on this situation. In practice the ultimate goal will be the possibility to predict the pressure drop vs flow rate relation from independent measurements of the rheological characteristics of the fluid and of the geometrical characteristics of the porous media (based on macroscopic parameters such as a permeability).

The existing approaches for establishing the pressure drop vs flow rate relation for yield stress fluids have relied on the usual physical approaches of the permeability coefficient appearing in the Darcy's law for Newtonian fluids: they use the analogy of the flow at a local scale within the porous medium with the flow either through a capillary or around isolated particles. Thus one can obtain an 
equation relating the pressure drop to the average velocity involving specific but partly unknown lengthscales (pore size, total flow path length). In this way various expressions for $\Delta p=f(V)$ have been obtained [8-12] which all have the following structure, typical of yield stress fluids: the velocity differs from zero only beyond a critical pressure drop $\Delta p_{c}$; then it progressively increases as the difference between the actual pressure drop and $\Delta p_{c}$ increases.

The relevance of these approaches for yield stress fluids is questionable. In particular Balhoff and Thompson [13] suggested that for each of these relationships "it is not yet clear whether universally applicable adjustements (in particular of the lengthscales) can be made or whether a case-by-case assessment is needed". Balhoff and Thompson [13] first evoke that the converging-diverging behavior of individual throats has a prominent effect for yield stress fluids.

In fact we emphasize that the essential point is that in contrast with the flow through a capillary, the flow of a yield stress fluid through a pore does not necessarily involve a constant size of the possible volume of fluid transported: as the pressure drop increases a wider region of fluid starts to flow. So, in contrast with Newtonian fluids, as the pressure drop increases the characteristic length related to the fluid volume available for flow at a local scale would increase. A second difference of flow characteristics is that at a macroscopic scale flow actually starts as a percolation effect: at the critical pressure drop the flow occurs only along a specific path throughout the porous medium [13-14]; then, we can expect that as the pressure drop is increased more flowing paths progressively form within the porous medium. In fact these effects should not lead to an apparent behavior at a macroscopic scale fundamentally different from that which has been observed or predicted: they are consistent with a critical pressure threshold and then an increase of pressure drop with velocity. The point is that the lengthscales involved in this law might not vary at all as expected in the above theoretical approaches.

Besides there have been several approaches based on network models [5,15] which provide more straightforward descriptions of the reality but don't provide generic analytical expressions for the pressure drop vs velocity relationship.

Unfortunately experimental studies in that field [8-10] are still scarce and rely on parameter variations (pore size or rheological parameters) in rather limited ranges. In addition existing data provide measurements of flow through porous medium in rather narrow ranges of mean velocity, typically slightly more than one decade. This implies that the comparison with a model can hardly be discriminatory. Also the comparison is done in linear scale so that the discrepancies at low flow rates are neglected, a critical point which precludes a relevant estimation of the critical pressure drop. At last the authors don't discuss whether the ranges of shear rates explored in both flow types (rheometry and flow through porous medium) are similar so as to support the relevancy of a comparison with the predictions of a model using the rheological parameters measured independently.

Actually a major difficulty in experiments of non-Newtonian fluid flow through porous media is to be able to obtain reliable data bases for both the rheological behavior of the fluid and its transport properties through the porous medium. As it is well known rheometrical tests with yield stress fluids need to be carried out carefully in order to obtain a relevant description of the material behavior [16] but on the basis of current knowledge it is possible to get reasonably relevant data. With an experiment of yield stress fluid flow transport through a porous medium various additional problems have to be taken into account. For example: standard pressure measurements cannot necessarily be used because of the development of normal stresses in cavities; additional pressure drop in the conduits before and after the porous medium may be significant for a yield stress fluid; the elasticity of the fluid in its solid regime may induce transient effects; the rheological properties of the fluid may be affected by its contact with the solid surfaces of the porous medium (ions or impurities) (a classical effect with Carbopol gels). 
We propose here an attempt to gather a set of relevant data for the two types of tests (rheometry and flow transport through porous media), for a wide range of shear rates and different sizes of porous media, different pore sizes and fluid yield stresses. With that aim, we used a set up in which the different possible experimental problems mentioned above are minimized. The wide data base obtained under such different conditions makes it possible to analyze the results in detail and get a general expression for the pressure drop vs flow rate, i.e. an empirical Darcy's law for yield stress fluids, with the appropriate scalings. After presenting the experimental set up, materials used and procedures in Section 2, we then present the data in Section 3, which are subsequently analyzed in Section 4.

\section{Materials and procedures}

\subsection{Materials}

In order to validate our measurement system, procedure and data analysis approach we carried out tests with a (Newtonian) Glycerol solution (99+\%, Alfa Aesar), exhibiting a viscosity of 1.5 Pa.s at $20^{\circ} \mathrm{C}$.

The first type of model yield stress fluid used was Carbopol (U980) solution in water. It has been observed that this material is essentially a glass made of a high concentration of individual, elastic sponges (with a typical element size of 2 to $20 \mu \mathrm{m}$ ) [17], which gives rise to its yielding behavior. The key advantage of this material is that it shows limited thixotropy [17-18] and phase migration, making it a quite well-behaved yield stress fluid. The preparation of Carbopol gel begins with the introduction of some water in a mortar mixer. The rotation velocity is set at $90 \mathrm{rpm}$ and the appropriate amount of raw Carbopol powder is slowly added to the stirring water. After about one hour, the incorporation of the powder is done and the appropriate amount of Sodium Hydroxide (1 $\mathrm{mol} / \mathrm{L}$ ) is quickly added to the solution, which increases its $\mathrm{pH}$. The mixing is then maintained for approximately one day to allow a full homogenization of the mixture. Such a procedure makes it possible to obtain a material which remains stable for several months. Different Carbopol concentrations were used, leading to different rheological properties. Concentrations from 0.1 to $0.8 \%$ were used but because of a significant influence of the actual mixing procedure, mixer type and mixing speed, on the solution finally obtained, we do not use, in the following, the concentrations as a parameter to identify a given solution but we rather rely on its rheological properties.

The second type of model yield stress fluid was water-in-oil emulsion obtained by dispersing in an oil phase droplets of brine stabilized by a surfactant. At high volume fraction of the dispersed phase, droplets come into contact and the fluid exhibits a yield stress [19]. Batches of water-in-oil emulsion are prepared by dispersing a water $+\mathrm{CaCl}_{2}(150 \mathrm{~g} / \mathrm{l})$ salt solution in a surfactant solution (dodecane (Acros Organics) $+7.5 \%$ wt of Span 80 surfactant) with a Silverson L4RT mixer. The rotation speed of the mixer is kept at $700 \mathrm{rpm}$ during the addition of brine. It is then increased up to $6000 \mathrm{rpm}$ during $85 \mathrm{~min}$ until a homogeneous emulsion is obtained. The droplet concentration was varied slightly around $80 \%$. In that case a slight evolution of the system (possibly some kind of coarsening) could be observed between some periods of tests. As a consequence, like for the Carbopol solutions these materials were basically characterized through their rheological properties. The droplet size was around $1 \mu \mathrm{m}$.

\subsection{Rheology}

Rheological tests were performed with a Bohlin-stress-controlled rheometer equipped with two circular, rough plates (diameter: $40 \mathrm{~mm}$ ). The sample was carefully set up and the gap was fixed at 2 $\mathrm{mm}$ taking care not to entrap air bubbles. A logarithmically increasing then decreasing stress ramp test was then applied over a total time of four minutes. Except for the first part of the increasing 
curve associated with deformations in the solid regime the increasing and decreasing shear stress vs shear rate curves almost superimpose. We retained, here, the decreasing part as the flow curve of the material. For similar Carbopol solutions and emulsions it has been shown that this apparent flow curve obtained from macroscopic observations correspond to the effective, local constitutive equation observed at a local scale with imaging technique [20].

Carbopol gels and emulsions exhibit a simple yield stress fluid behaviour and their flow curve can be well fitted by a HB (Herschel-Bulkley) model (see typical results in Figure 1):

$\tau>\tau_{c} \Rightarrow \tau=\tau_{c}+k \dot{\gamma}^{n}$

in which $\tau$ is the shear stress, $\dot{\gamma}>0$ the shear rate, $\tau_{c}$ the yield stress, $k$ the consistency factor and $n$ the power law exponent. For the different Carbopol solutions the material parameters were found in the following ranges: $8<\tau_{c}<89 \mathrm{~Pa}, 3.6<k<49 \mathrm{~Pa} . \mathrm{s}^{n}$ and $n=0.36$. For the emulsions we found: $54<\tau_{c}<71 \mathrm{~Pa}, 12.7<k<13.4 \mathrm{~Pa}^{n} \mathrm{~s}^{n}$ and $n=0.36$. Note that since the Herschel-Bulkley model contains three adjustable parameters one can find slightly different values depending on the fitting procedure used. As in our case we observed that the resulting value for $n$ ranged from 0.34 to 0.38 , we decided to fix its value to 0.36 for all materials and adjusted the two other parameters, which makes it possible to represent the data as accurately as through various other procedures.

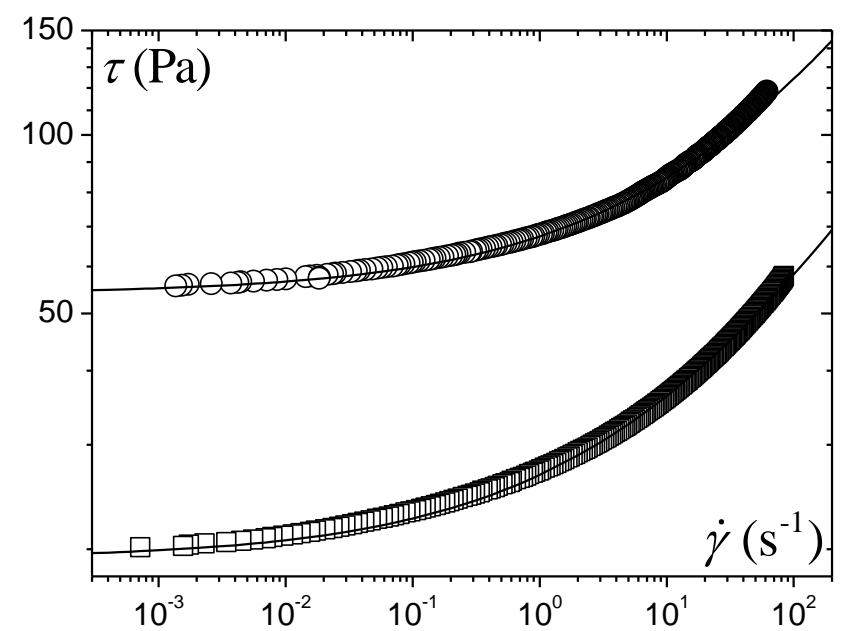

Figure 1: Typical flow curve of a Carbopol solution (squares) and an emulsion (circles). The continuous lines correspond to the HB model fitted to data with the following parameters: (Carbopol) $\tau_{c}=19.3 \mathrm{~Pa}, \quad k=7.4{\mathrm{~Pa} . \mathrm{s}^{n}}^{n} \quad$ and $\quad n=0.36 ; \quad$ (emulsion) $\tau_{c}=54 \mathrm{~Pa}, \quad k=13.4 \mathrm{~Pa} . \mathrm{s}^{n}$ and $n=0.36$.

\subsection{Experimental set up}

The setup used for carrying out the experiments has been adapted from a set of experiments initially developed for studying the injection of micro-cement suspensions into sands, taking into account the filtration and clogging phenomena [21-23], with application to the in situ improvement of mechanical properties of soils using grouting/injection processes. The set up is made up of two main components, (see Figure 2) which are: (i) an injection setup composed of a two-part (oil/fluid) injection reservoir, connected to an air/oil interface cell allowing to apply a regulated oil pressure in the reservoir; (ii) a confinement cell which contains the confined porous medium. This cell is connected, through plastic tubings, to the injection reservoir. The experimental setup is 
instrumented with pressure transducers (measurement of oil an fluid pressures during the test) and with a precision weigh scale installed at the exit of the confinement cell (see figure 2.a) for continuously recording the mass of fluid flowing out of the porous medium and calculate the flow rate.

The injection reservoir (see Figure 2a) is composed of two parts (bottom and top parts) separated by a low stiffness waterproof "Bellofram" membrane. The reservoir is $15 \mathrm{~cm}$ in diameter and $36 \mathrm{~cm}$ in height. The bottom part of the reservoir is filled up with oil (Newtonian fluid). This part is connected to the air-oil interface cell, which makes it possible to control the injection pressure. A pressure transducer is placed at the oil outlet point. The latter measures the relative oil pressure, $p_{i}$, with respect to the atmospheric pressure. The increase of oil volume in the bottom part of the reservoir induces a displacement of the membrane which, in turn, pushes the fluid to be injected, located in the upper part of the reservoir, out of the reservoir. The fluid flowing out is then channeled to the confinement cell and injected in the porous medium. We believe that the present technique used for measuring the injection pressure, via the upstream pressure in a Newtonian fluid (the oil), makes it possible to avoid possible errors which could occur if placing a pressure transducer directly in contact with the yield stress fluid.

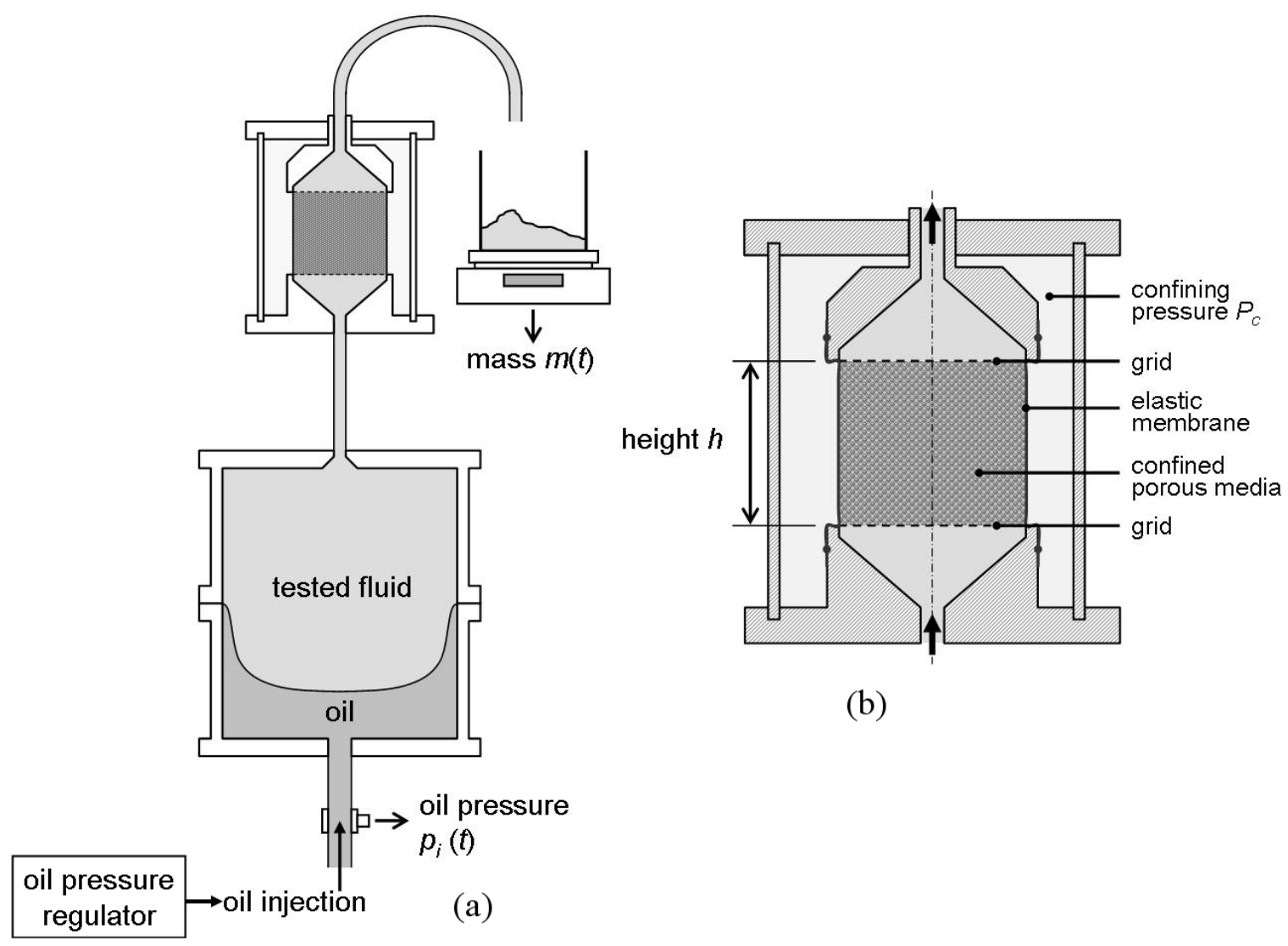

Figure 2: Functional scheme of the complete set up used for injection (a) and simplified cross-section of the confinement cell (b).

A simplified cross-section of the confinement cell, showing the porous sample, is presented in Figure $2 \mathrm{~b}$. The porous medium is contained in a thin waterproof latex membrane which allows the application to the specimen, through the water filling up the confinement cell, a confining pressure Pc. Metallic grids are placed at the top and bottom of the specimen in order to retain the granular material (beads) during the test. The diameter of the specimen is $8 \mathrm{~cm}$, different heights may be used $(4,8$ or $16 \mathrm{~cm})$. In order to prevent the development of preferential paths and obtain a uniform 
flow entering the specimen, the latter is placed on a conical hollow bottom plate. A similar conical end plate is adjusted on top of the specimen for the outgoing fluid.

The fluid flowing out of the sample falls into a small reservoir set on the precision balance mentioned before (Figure 2.a). We checked that the tested fluid did not evolve during the test by sampling it at the exit tube and comparing its rheological properties with those of the injected fluid.

\subsection{Porous medium}

We used different porous media made up of glass bead packings. Different types of beads were used, with a mean diameter $(D)$ : ranging from 0.26 to $2 \mathrm{~mm}$. We measured the size distribution of the beads with a laser granulometer (Laser Beckman-Coulter). Typical sets of data obtained with this technique are shown in Figure 3. It appears that the size distributions of the beads are almost perfectly similar. A more detailed analysis of these distributions can be carried out with the help of the polydispersity coefficient defined as $P=100 \sum \phi_{i}\left|D-d_{i}\right| / D \sum \phi_{i}$ where $\phi_{i}$ is the volume fraction of the beads having a diameter $d_{i}$. We found that $P$ varies between 8 and $12 \%$ depending on the grain type. This confirms that our grains are almost monodisperse with a very similar polydispersity, which justifies that in the following we consider that we have a single type of porous medium, i.e. the different porous media are similar and characterized by only one length scale, namely the mean diameter $D$.

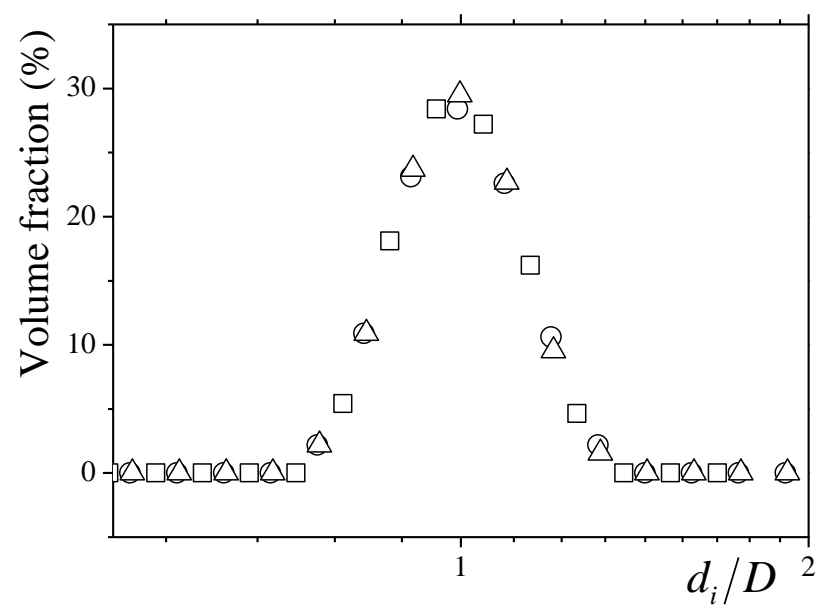

Figure 3: Laser granulometry data for glass beads with three different mean diameters $(D): 0.26 \mathrm{~mm}$ (triangles), $0.6 \mathrm{~mm}$ (circles), $1.1 \mathrm{~mm}$ (squares).

As some impurities remaining on the bead surface may destabilize Carbopol solutions we tested the impact of cleaning the beads in an ultrasonic bath during one hour. The data obtained with or without such cleaning were similar.

The porous medium is made by slightly compacting, manually, successive layers of beads in a forming mould against which is initially applied the latex membrane. The porosity finally obtained is $0.34 \pm 6 \%$, which in particular means that our porous medium preparation was reproducible. Then, a confining pressure $P_{c}=100 \mathrm{kPa}$ is applied to the porous medium, which moulds the membrane onto the beads and maintains the grain packing. It is expected that this technique limits 
the development of preferential flow paths at the periphery of the porous medium, a phenomenon which tends to occur when the bead pack is in contact with a planar surface. Indeed, in the latter case, the porosity is larger close to the planar surface than inside the porous medium. Note that we did not observe any significant compaction or widening of the sample section due to the confinement.

In order to calibrate the system for head losses due to the equipement itself (calibration for system compliance) and to be able to make corresponding corrections on the raw data obtained, specific tests were carried out by replacing the porous medium by a rigid empty tube that was called the phantom. The phantom, equipped, inside, with a latex membrane similar to the one used for the real test, had the same inner diameter as the porous medium. The cylinder was then externally covered with another latex membrane, allowing to adjust the phantom with the bottom and top end plates of the confinement cell.

\subsection{Experimental procedure}

We first slowly introduce the fluid in the top part of the injection reservoir. For yield stress fluids this step is crucial in order to limit the number of air bubbles introduced into the reservoir during this operation. Then the porous medium is prepared as described above. The injection reservoir is then connected to the confinement cell. Afterwards the impregnation of the porous medium can start: the oil pressure is increased to reach a pressure gradient of approximately 20 times a critical pressure gradient (estimated from the fluid yield stress divided by the bead's mean diameter). Such a pressure a priori makes it possible to saturate the porous medium in a short time with a sufficiently slow flow rate. The process is stopped when the flow observed on the exit tube of the confinement cell is well established. At that time the sample is supposed to be fully saturated. Note that at any time during the test it is necessary to check that the confining pressure is significantly larger than the oil pressure in order to maintain the rigidity of the bead packing.

We then start to increase the pressure step by step until reaching a continuous flow at the exit. Then the pressure is decreased step by step. This decrease is stopped when the out-flow does not seem to stabilize, typically for a flow rate less than $0.3 \mathrm{drop} / \mathrm{min}$. For each pressure level, the measurement is initiated after stabilisation of the flow as observed on the exit tube, and this pressure is maintained during a sufficient time to get a measurable mass evolution at the exit. For example, for flow rates as low as a few drops per minute we wait for at least 10 drops going out of the device. Note that a different bead pack was used for a test with each new fluid, i.e. after a series of measurements at different velocities with one fluid type the beads were thrown away.

\section{Experimental results}

3.1 Procedure for analyzing the data

A typical data set for $p_{i}(t)$ and $m(t)$ is shown in Figure 4. These data can be analyzed in terms of pressure drop per unit length, i.e. $\nabla p=p_{i} / h$ in which $h$ is the height of the porous medium, vs the flow rate, i.e. the measured (average) velocity of the material through the porous medium:

$V=\frac{1}{\rho S} \frac{\Delta m}{\Delta t}$

in which $S$ is the section area of the porous medium and $\rho$ the fluid density. Note that the above definition for the pressure drop will find a full physical meaning only when we have computed 
the effective pressure drop associated with the flow through the porous sample only (see below). A typical apparent flow curve, i.e. $\nabla p$ vs $V$, obtained in this way is shown in Figure 5.

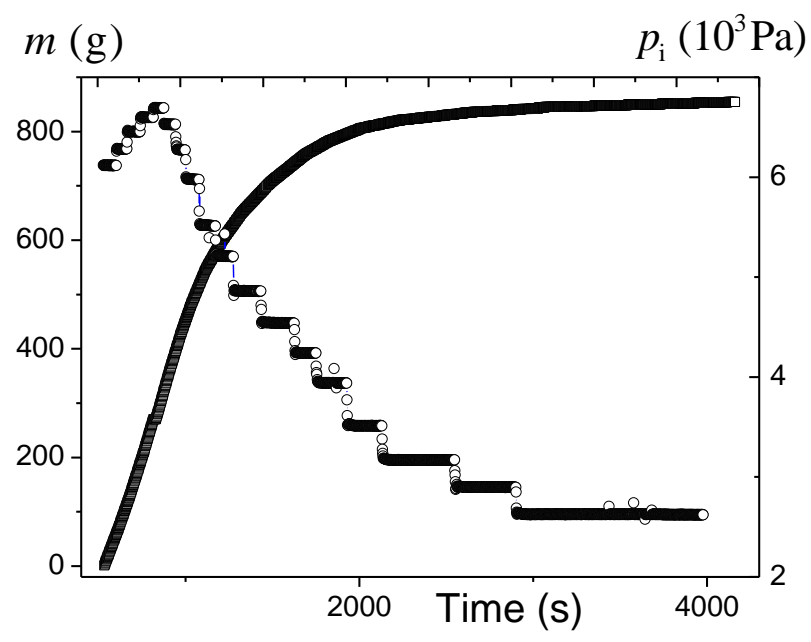

Figure 4: Typical set of data for a complete test of injection (bead size $0.26 \mathrm{~mm}$, (Carbopol) $\tau_{c}=78 \mathrm{~Pa}, k=33 \mathrm{~Pa} . \mathrm{s}^{n}$ and $n=0.36$ ) including a pressure (circles) increase step by step followed by a pressure decrease, and the corresponding mass evolution in time (squares).

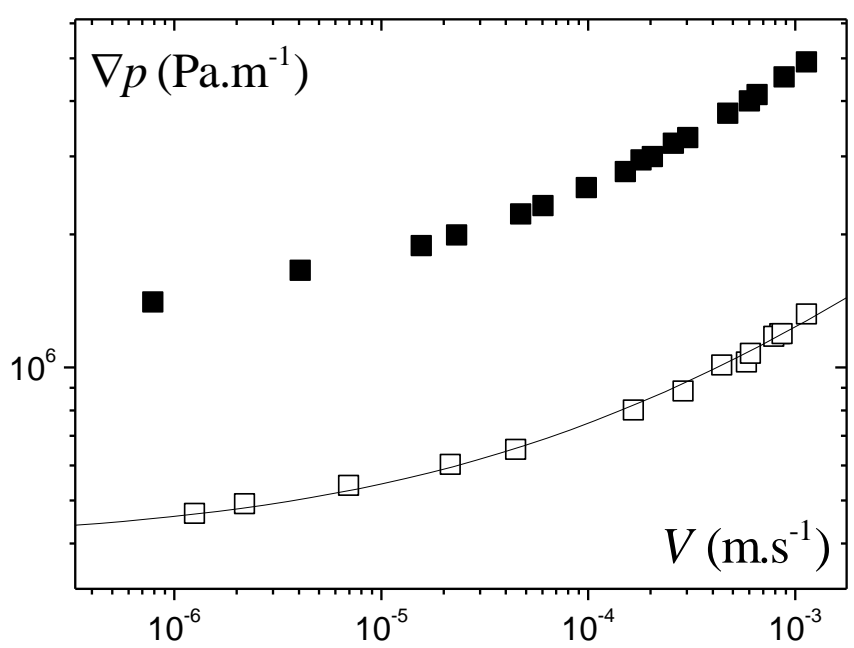

Figure 5: Typical interpretation of the mass and pressure data in terms of pressure gradient and flow rate for an injection test of the type shown in Figure 4 (filled squares), and for the same test with the phantom (open squares). The continuous line is a HB model fitted to phantom data (see text).

For each injection test with beads we also carry out a calibration test with the phantom, and analyzed the data the same way. We thus obtain two apparent flow curves (see Figure 5). In each case the pressure drop includes different pressure drops due to the flow through the different parts of the system except the porous medium. It also includes the hydrostatic pressure drop. These contributions are a priori the same when one uses a porous medium or a phantom. These calibration corrections allow us to obtain the effective flow curve, i.e. strictly associated with the flow through the porous medium (and without hydrostatic contribution), by subtracting the apparent flow 
curve associated with the phantom data from the flow curve found with the porous sample. Such a procedure in particular makes it possible to get relevant data without having to measure the pressure in the yield stress fluid (for example at the inlet and outlet of the confinement cell). This operation would indeed be difficult and not necessarily reliable because the pressure is not well defined in a yield stress fluid at rest. Note that in practice in order to facilitate the subtraction we first fitted a model to the flow curve data for the phantom (see Figure 5).

We observed flow rates in the range $10^{-6}-10^{-3} \mathrm{~m} \cdot \mathrm{s}^{-1}$. Estimating the typical shear rate in the porous medium from the ratio of the effective mean velocity through the porosity to the typical pore size (around one third the bead radius) we find that in all our tests it varied in the range $10^{-2}-10^{2} \mathrm{~s}^{-1}$. This corresponds to the range of shear rates covered in our rheometrical tests.

\subsection{Results for Glycerol}

Injection tests were carried out with Glycerol for two bead sizes ( $1 \mathrm{~mm}$ and $2 \mathrm{~mm}$ ). After subtracting the (constant) hydrostatic pressure term the flow curve obtained for the phantom appears to be linear (see Figure 6). This is the typical result obtained for the flow through a conduit or a porous medium. This indicates that in our range of measurements the whole system around the bead packing behaves like a given porous medium, which supports our procedure for extracting the effective flow curve (see above).

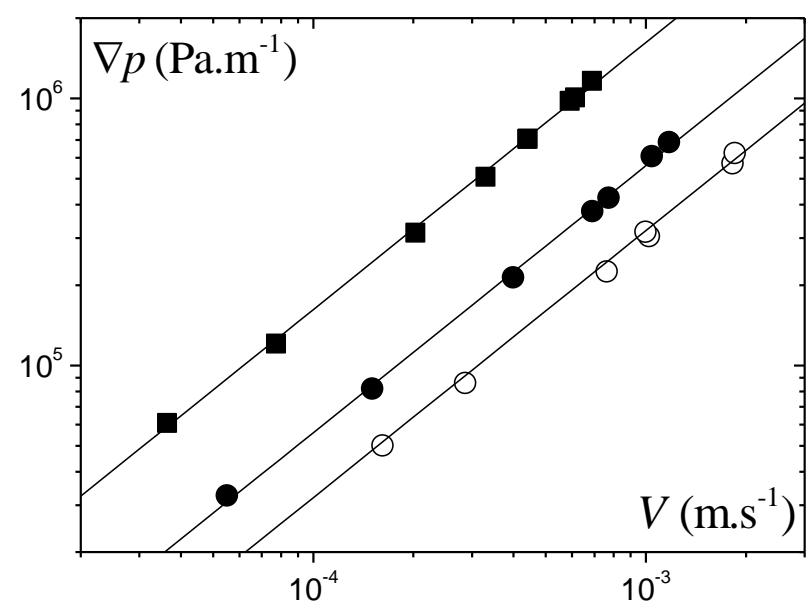

Figure 6: Glycerol injection tests: apparent flow curve for the phantom (after subtracting the hydrostatic pressure drop) (open circles) and effective flow curves for two porous media: $D=1.1 \mathrm{~mm}$ (filled squares), and $D=2 \mathrm{~mm}$ (filled circles). The continuous lines are linear fits of these data.

The effective flow curves obtained for the Glycerol solutions through the porous media are also linear (see Figure 6), in agreement with Darcy's law:

$\nabla P=\frac{\mu}{K} V$

in which $K$ is the permeability of the medium. Since the porous media are similar (see Section 2.4) except for their characteristic length $(D)$ we deduce from scaling arguments that the permeability should be proportional to $D^{2}: K=\alpha D^{2}$. From our data (linear fits of Figure 6) we find for $\alpha$ $7.8 \times 10^{-4}$ for $D=1.1 \mathrm{~mm}$ and $6.7 \times 10^{-4}$ for $D=2 \mathrm{~mm}$. This globally confirms the validity of our approach but shows that the results are not perfect, which appears reasonable considering the 
uncertainties at the different steps of the process (phantom, slight viscosity variations, slight differences in the porous media, etc). Note that the empirical Kozeny-Carman equation [24] for the same porosity predicts a slightly smaller value $\left(\alpha=5 \times 10^{-4}\right)$ than the above one, which may be due to some difference in the grain size distribution.

It is worth recalling that for a liquid flowing through a straight cylindrical conduit the flow curve is formally similar to (3) but now with $K=\alpha^{\prime} D^{2}$ and $\alpha^{\prime}=3.1 \times 10^{-2}$. This means that the equivalent diameter of a conduit which would represent the porous medium in terms of Darcy's law for a Newtonian fluid is equal to $d=\sqrt{\alpha / \alpha^{\prime}} D \approx D / 6.6$. The value of the final factor, i.e. 6.6, between the bead diameter of the porous medium and the diameter of the equivalent conduit in terms of Darcy's law mainly results from the three following effects: (i) an average thickness of the paths between the beads significantly smaller than the bead diameter; (ii) a section area available for fluid smaller than the apparent section due to the presence of the beads, which increases the effective velocity through the equivalent conduit; (iii) the tortuosity of the fluid paths which tends to increase the length of these paths.

\subsection{Results for yield stress fluids}

The apparent flow curves for the phantom have a shape similar to the flow curve of the material obtained from rheometrical tests. Under these conditions we decided to fit these curves with a Herschel-Bulkley model with the same exponent as that used for fitting the flow curve (i.e. $n=0.36$ ).

The resulting effective flow curves for the different materials and mean bead diameters all present the same shape: they exhibit a plateau at low velocities and the pressure drop progressively increases with increasing velocity. This shape is similar to the typical (rheometrical) flow curve of a simple yield stress fluid. This means that in order to induce a flow through a porous medium one needs to overcome a critical pressure drop.

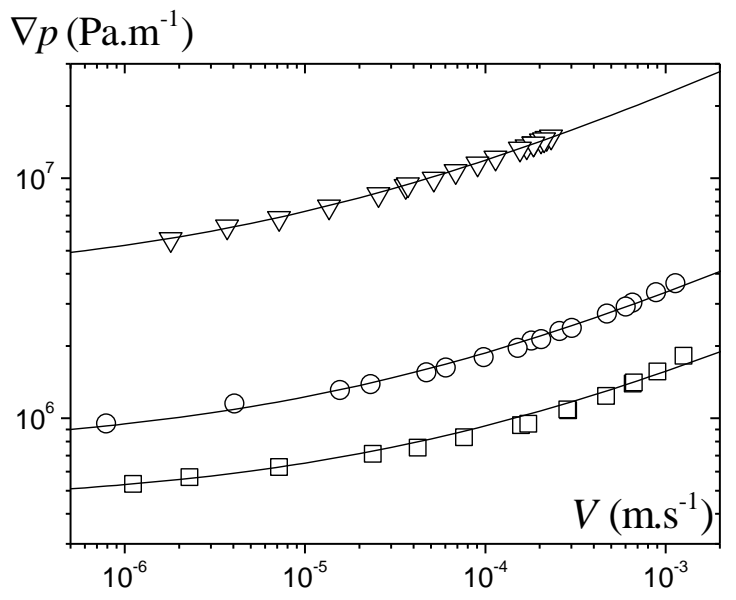

Figure 7: Effective flow curves for injection tests with a Carbopol solution $\left(\tau_{c}=78 \mathrm{~Pa}, k=33 \mathrm{~Pa} . \mathrm{s}^{n}\right.$ and $n=0.36$ ) through different porous media: $D=2 \mathrm{~mm}$ (squares), $D=1.1 \mathrm{~mm}$ (circles) and $D=0.26 \mathrm{~mm}$ (triangles). The continuous lines correspond to fits of the Herschel-Bulkley model to the data (see text).

When the bead size decreases or when the material yield stress increases the shape of the flow curve does not seem to change significantly (see Figures 7 and 8): at first sight it seems that this is essentially the critical pressure drop which increases while the additional velocity dependent 
component is approximately constant (the curves are roughly similar by vertical shift in a logarithmic scale). The more detailed analysis presented below will show that this is not always right: the velocity-dependent component also significantly varies when the pore size varies. Anyway the vertical shift (in logarithmic scale) of the flow curves for a decreasing pore size or an increasing yield stress globally corresponds to the expected impact of the permeability decrease or the viscosity increase as for any type of viscous fluid flowing through any type of porous medium.

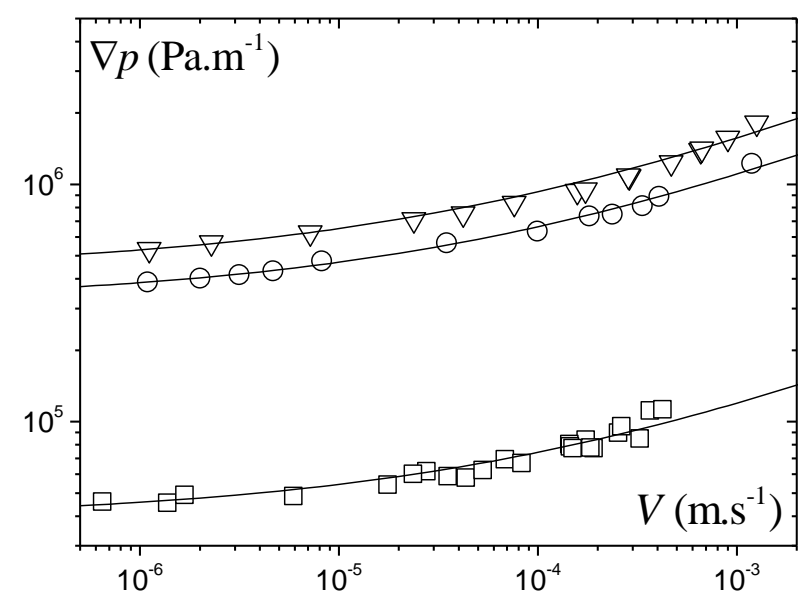

Figure 8: Effective flow curves for injection tests through a porous medium $(D=2 \mathrm{~mm})$ with different Carbopol solutions: $\tau_{c}=8 \mathrm{~Pa}, \quad k=3.6 \mathrm{~Pa} . \mathrm{s}^{n}, \quad n=0.36$ (squares); $\tau_{c}=55 \mathrm{~Pa}$, $k=23.5$ Pa.s ${ }^{n}, n=0.36$ (circles); $\tau_{c}=78 P a, k=33$ Pa.s ${ }^{n}, n=0.36$ (triangles). The continuous lines are fits of the Herschel-Bulkley model to the data (see text).

Let us now compare the results obtained for Carbopol solutions and emulsion having similar rheological properties and flowing through the same porous medium. Surprisingly the effective flow curves are significantly different (see Figure 9). In particular the critical pressure drop for the emulsion is significantly lower than for that for the Carbopol solution by a factor of about two. This difference cannot be explained solely by the difference of values of the parameter $k$ for the two types of fluid. This suggests that there is an unexpected impact of the nature of the fluid, a point that we will discuss further below.

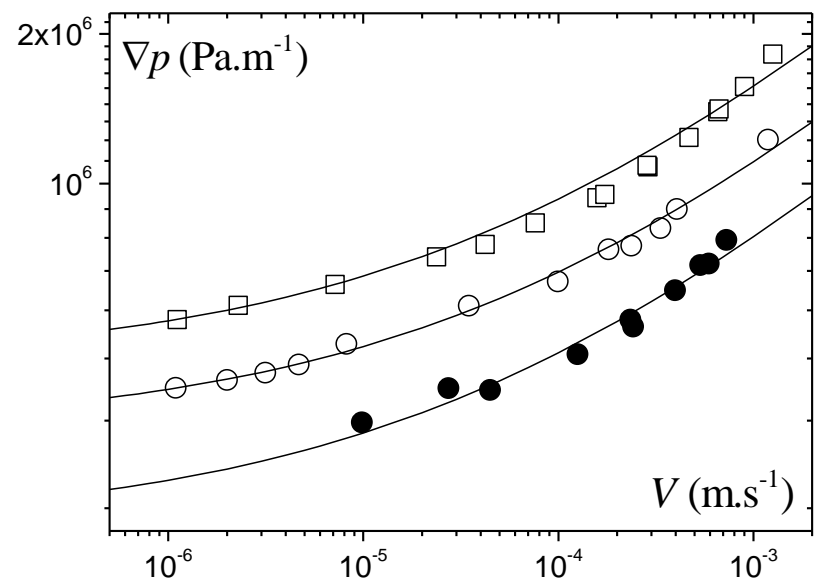


Figure 9: Effective flow curves for injection tests through a porous medium ( $D=2 \mathrm{~mm}$ ) with two Carbopol solutions: (open circles) $\tau_{c}=55 \mathrm{~Pa}, k=23.5 \mathrm{~Pa} . \mathrm{s}^{n}, n=0.36$, and (squares) $\tau_{c}=78 \mathrm{~Pa}$, $k=33$ Pa.s ${ }^{n}, n=0.36$; and an emulsion: (circles) $\tau_{c}=71 \mathrm{~Pa}, k=12.7 \mathrm{~Pa} . \mathrm{s}^{n}, n=0.36$. The continuous lines are fits of the Herschel-Bulkley model to the data (see text).

Considering the shape of the effective flow curves for all the injection tests it is quite natural to fit a Herschel-Bulkley model to the data keeping the same exponent as in the fit on rheometrical data. This model here expresses as:

$\nabla P=\nabla P_{c}+k_{p} V^{n}$

in which $\nabla P_{c}$ is the critical pressure drop and $k_{p}$ the consistency parameter for injection. This step is important as it means that it will now be possible to fully analyze the results simply through the values of the two parameters of this model as a function of the experimental parameters (bead diameter, fluid yield stress and consistency, flow rate).

In a first step we can now plot the two injection parameters as a function of the corresponding rheological fluid parameters. Globally each parameter increases when the other one increases but it is clear that they is no straightforward exclusive relationship between them. The data representing the pressure drop as a function of the yield stress for all our experimental conditions are widely dispersed.

\section{Discussion}

\subsection{Dimensional analysis}

A simple comparison of the HB model parameters for injection and rheometrical tests is not relevant. Indeed $\nabla P_{c}$ and $k_{p}$ also depend on the characteristics of the porous medium through which the fluid flows. In order to take this aspect into account we can again take advantage of the fact that the porous medium is characterized by a single lengthscale, i.e. D. From a dimensional analysis we readily deduce that we should expect two relations in the form:

$$
\begin{aligned}
& \nabla P_{c}=\alpha \frac{\tau_{c}}{D} \\
& k_{p}=\beta \frac{k}{D^{n+1}}
\end{aligned}
$$

where $\alpha$ and $\beta$ are two unknown parameters which are independent of the material type and porous medium characteristics.

In order to check the validity of this analysis we plotted the data in the form suggested by equations (5) and (6). From this representation we can conclude that $\nabla P_{c}$ is indeed proportional to $\tau_{c} / D$ and $k_{p}$ is proportional to $k / D^{n+1}$ (see Figure 10): all the data obtained for each material fall along a straight line of slope 1 on a logarithmic scale. For $k_{p}$ a single straight line is obtained for the data corresponding to both materials, but for $\nabla P_{c}$ two different straight lines are obtained. This is consistent with our above observation, i.e. the injection flow curves obtained for an emulsion and for a Carbopol solution with similar yield stress were significantly different. Finally our results can be summarized in terms of a general model giving the pressure drop as a function of the flow rate, i.e. the equivalent of the Darcy's law for yield stress fluids: 
$D \nabla p=\alpha \tau_{c}+\beta k\left(\frac{V}{D}\right)^{n}$

In this expression $\alpha$ and $\beta$ should be universal factors for a porous medium composed of an assembly of spheres, and $V / D$ is the apparent shear rate for the flow through such a porous medium. The first (yielding) term on the right hand side of equation (7) corresponds to the critical pressure for incipient flow. As usual the second term, which is velocity dependent, derives from the additional viscous stress above the yield stress as long as the flow has been initiated.

The differences observed for the values of $\alpha$ for the two types of fluids would imply that they do not see the same porous medium, which is obviously not the case. Therefore, from a general point of view we need to understand the origin of the values for $\alpha$ and $\beta$ and it is expected that this understanding will provide some elements of explanation for the discrepancy found between the two fluids.
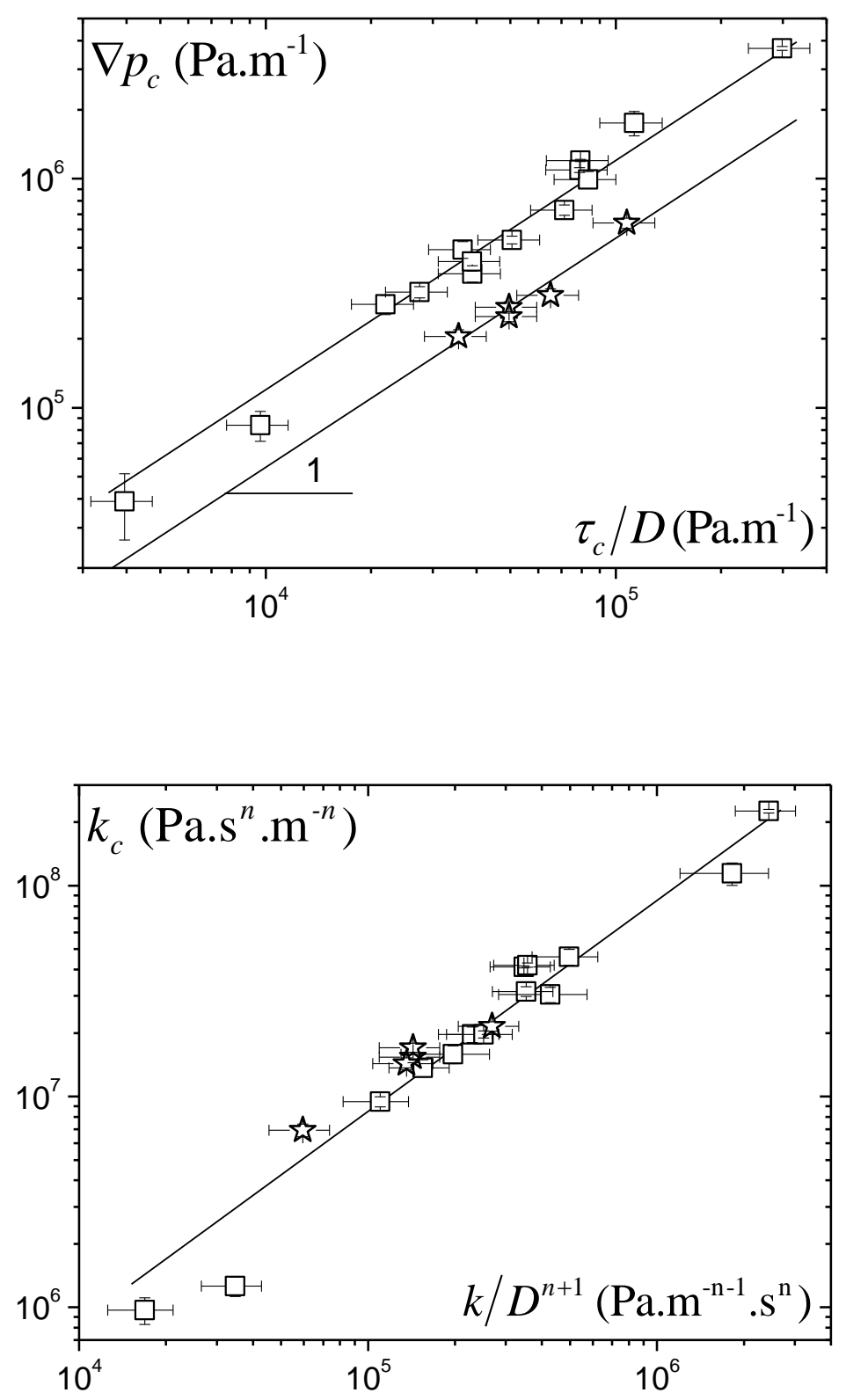
Figure 10: Parameters (critical pressure drop (a) and consistency (b)) of the Herschel-Bulkley model (equation (4)) fitted to data of injection tests as a function of the appropriate expressions deduced from dimensional analysis and involving the corresponding rheological parameters of the fluids for Carbopol solutions (squares) and emulsions (stars) and the porous medium characteristics. The continuous lines correspond to straight lines of slope $\alpha$ equal to 12 for the Carbopol and 5.5 for the emulsion in (a), and of slope $\beta=85$ in (b).

\subsection{Analogy with the flow through a simple conduit}

In order to further analyze our results it is useful to compare them with the flow characteristics for a flow through a straight conduit. Let us consider the steady uniform flow of a Herschel-Bulkley fluid in a cylinder of radius $R$. The momentum equation can easily be solved to obtain the mean velocity (flow discharge per unit surface) as the function of the pressure gradient (see for example [25]). This may be expressed in terms of the relationship between two dimensionless numbers, namely $G=R \nabla p / 2 \tau_{c}$ and $B i=\tau_{c} R^{n} / k V^{n}$ :

$G \leq 1 \Rightarrow B i^{-1}=0 ; G>1 \Rightarrow B i^{-m}=G^{-3}(G-1)^{m+1}\left[\frac{(m+1)(m+2) G^{2}+2(m+1) G+2}{(m+1)(m+2)(m+3)}\right]$

in which $m=1 / n$ and $\nabla p=\Delta p / L$ where $L$ is the conduit length. An explicit analytical expression for the pressure gradient as a function of the flow rate cannot be found from this equation. However we can deduce the expressions in the asymptotic cases:

$$
\begin{aligned}
& B i \rightarrow \infty \Rightarrow \nabla p=\frac{2}{R} \tau_{c} \\
& B i \rightarrow 0 \Rightarrow \nabla p=2(m+3)^{n} \frac{1}{R} k\left(\frac{V}{R}\right)^{n}
\end{aligned}
$$

Note that the expression (9a) could be obtained directly from a simple momentum balance for describing the incipient motion of the yield stress fluid through the conduit. On another hand the expression (9b) is simply the solution of the flow problem with a power-law fluid.

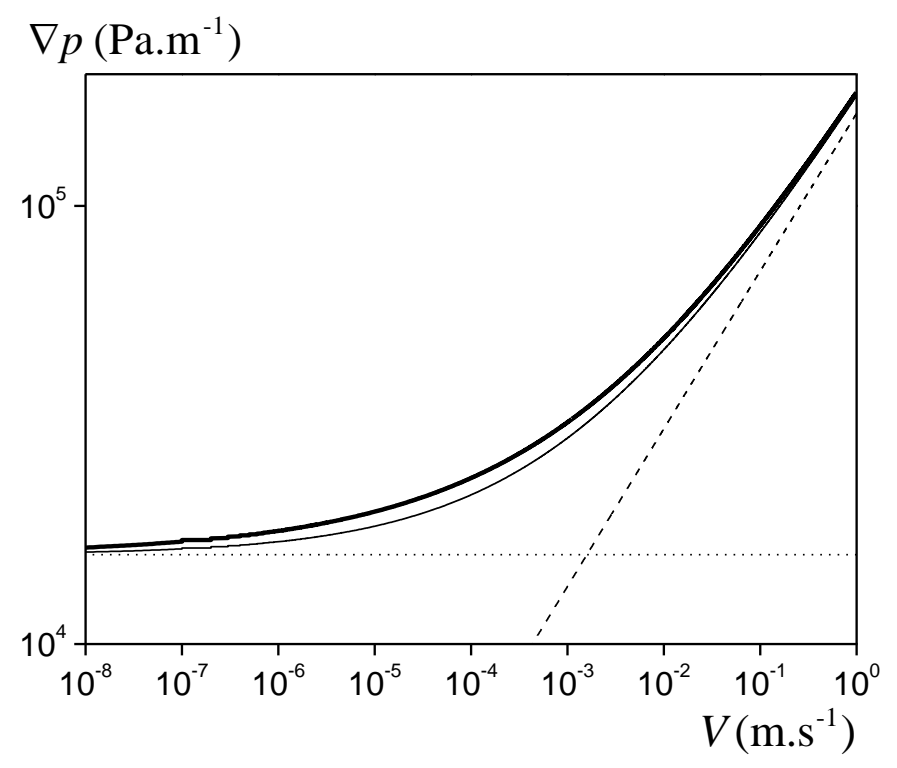

Figure 11: Different models for representing the pressure drop vs velocity for the steady flow of a yield stress fluid ( $n=0.36, \tau_{c}=8 \mathrm{~Pa}, k=3.6 \mathrm{~Pa} . \mathrm{s}^{\mathrm{n}}$ ) through a cylindrical conduit (of radius $R=1 \mathrm{~mm}$ ) : critical pressure for incipient motion (dotted line), viscous term in the absence of 
yielding (dashed line), sum of the two previous terms (thin continuous line). The exact theory

(according to equation (8)) is represented by the thick continuous line.

An approximate expression can naturally be found by adding the two asymptotic terms above and we obtain:

$d \nabla p=\alpha^{\prime} \tau_{c}+\beta^{\prime} k\left(\frac{V}{d}\right)^{n}$

in which $d=2 R, \alpha^{\prime}=4$ and $\beta^{\prime}=4(6+2 / n)^{n}$. This expression appears to well represent the theoretical curve within $10 \%$ over the whole range of $B i$ (see Figure 11).

It is instructive to compare the values obtained for Darcy's law for the porous medium to those corresponding to the flow through this simple conduit. With (10) we have an expression equivalent to that obtained from our experiments for the flow through a porous medium so that we can find directly the conduit size which would lead to the same pressure drop vs flow rate expression. Let us first consider the yielding term, i.e. $\alpha \tau_{c}$. For the Carbopol solution and the emulsion we find values (see Figure 10a) which lead to a ratio between the bead and the conduit diameters which are respectively 1.4 and 3 . These values significantly differ from the factor (6.6) corresponding to the Newtonian Darcy's law (see Section 3.2), which suggest that there is no simple analogy between the physical mechanisms in the two situations.

Let us now further examine the possibility of equivalence between the two types of flow. This may be done by remarking that for the flow through a simple conduit the ratio of the two factors is constant, i.e. independent of velocity, rheological parameters and conduit size: $\alpha^{\prime} / \beta^{\prime}=0.41$. For the flow through a porous medium we find $\alpha / \beta=0.14$ for Carbopol solutions and $\alpha / \beta=0.065$ for emulsions. This clearly means that, although it gives reasonable values for the critical pressure drop, the equivalence between the simple conduit flow and a porous medium for a yield stress fluid is not consistent.

\subsection{Analogy with the flow through a bundle of conduits}

In order to understand the origin of the above observed inconsistency we look at the flow characteristics of a yield stress fluid through a bundle of parallel conduits of different sizes. We consider a set of $N$ conduit of widths $\alpha_{i} R(i=1, \ldots N)$. The number of conduits per size $\left(n_{i}\right)$ is inversely proportional to their section area so that the total area for each conduit type is constant. All the conduits are parallel to each other, so that the applied pressure drop is given and independent of the conduit size. Under such conditions, as we increase the pressure drop the flow is initiated in the conduits of largest size while the fluid remains in its solid regime elsewhere. Then as the pressure drop is increased the flow begins in the slightly smaller conduit and so on.

We define a specific dimensionless pressure drop for each conduit radius: $G(i)=\alpha_{i} G$, in which $G$ is the value for the largest conduit. The flow in each conduit type is governed by the value of $G(i)$ according to: $G(i) \leq 1 \Rightarrow x_{i}=0 ; G(i)>1 \Rightarrow x_{i}=1$. From the mean velocity through each conduit (8) we deduce the mean velocity through the set of conduits: 
$G<1 \Rightarrow B i^{-1}=0 ; G>1 \Rightarrow B i^{-m}=\frac{G^{-3}}{\sum_{i} \alpha_{i}^{2} n_{i}}\left[\frac{\sum_{i} n_{i} x_{i}\left(\alpha_{i} G-1\right)^{m+1}\left((m+1)(m+2) \alpha_{i}^{2} G^{2}+2(m+1) \alpha_{i} G+2\right)}{(m+1)(m+2)(m+3)}\right]$

in which $B i$ is defined the same way as above, based on the radius of the largest conduit.

Finally the pressure drop vs velocity curve has the usual form with a plateau associated with the critical pressure drop for flow to occur in the first conduit and a finite slope for large velocities. This slope should tend to 1 for very large velocities when the fluid flows in all of the conduits. As a consequence we can still use the approximation of the sum of the two asymptotic expressions for the pressure drop (as in (10)):

$G=1+(m+3)^{1 / m} B i^{-1}\left(\sum_{i} \alpha_{i}^{2} n_{i} / \sum_{i} \alpha_{i}^{m+3} n_{i}\right)^{1 / m}$

With such a form the characteristics of the set of conduits is accounted for by a single coefficient multiplied by $B i^{-1}$. This approximation is slightly not as good as for the single conduit, the maximum error being $15 \%$ for $n=0.36$ in the transition region, but still constitutes a useful relevant representation. Finally this gives an expression equivalent to (10) for the pressure drop with now:

$\alpha^{\prime}=4$ and $\beta^{\prime}=4(m+3)^{1 / m}\left(\sum_{i} \alpha_{i}^{2} n_{i} / \sum_{i} \alpha_{i}^{m+3} n_{i}\right)^{1 / m}$

The origin of this new value for $\beta^{\prime}$ is that an increasing amount of fluid flows when the pressure drop increases. We computed the value of the ratio $\alpha^{\prime} / \beta^{\prime}$ for a range of one hundred conduit radii distributed in a logarithmic way between the maximum value $d$ and the minimum value $\chi d$ (see Figure 12). We see that widening the range of diameters induces, indeed, a decrease of the ratio $\alpha^{\prime} / \beta^{\prime}$. This is not sufficient to reach a value as low as that observed for the emulsion (0.065) but it appears possible to reach an appropriate value by using another arbitrary type of size distribution, in particular by taking into account a higher fraction of lower diameter conduits.

Finally we can conclude that the first coefficient in the pressure drop equation, i.e. the equivalent of Darcy's law for yield stress fluid, mainly finds it physical origin in the widest path through the medium, while the second coefficient finds its origin in the complex multiscale flow through the medium. As a consequence the basic origin of the relatively low value of the coefficient $\beta$ is the fact that in a porous medium the widest fluid path is first mobilized for the critical pressure drop while thinner and thinner paths, and thus smaller and smaller pores are then mobilized for increasing pressure drop. The next step will be to discuss the origin of the different values found for $\alpha$. The curious point is that we have a consistency of data through the general scaling laws with regards to a continuum approach, whereas these laws differ for two materials with different internal structures but the same mechanical properties. 


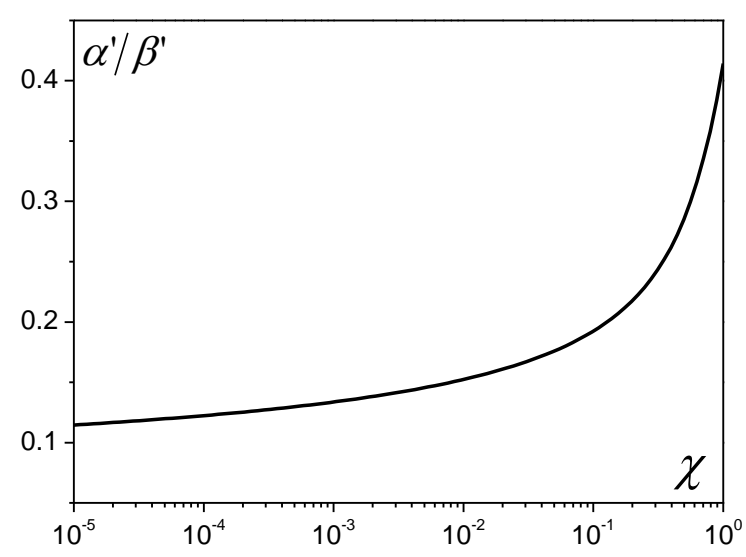

Figure 12: Ratio of the two coefficients of equation (12) as a function of the minimum to maximum diameter ratio for the flow through a series of conduits of different diameters (see text).

\subsection{Flow through sieves}

In order to understand the origin of the discrepancy between the values of $\alpha$ associated with the critical pressure drop for the two different materials we carried out tests consisting to leave the material flow through a simpler porous medium, i.e. a sieve, now under the action of gravity only. The experimental device is composed of a plexiglass tube $(30 \mathrm{~cm}$ long and $8 \mathrm{~cm}$ wide) over a sieve with a mesh size ranging from $20 \mu \mathrm{m}$ to $2 \mathrm{~mm}$. The set-up is weighed during the flow in order to obtain the mass of fluid percolating through the sieve.

Figure 13 shows a comparison of the results obtained for a Carbopol solution with a yield stress of $65 \mathrm{~Pa}$, and an emulsion with a yield stress of $76 \mathrm{~Pa}$, for two different sieve sizes. The mass evolves by jumps because the fluid going through the sieve progressively accumulates just below it and ultimately falls when it has reached a critical mass value. This resembles the behavior of a simple liquid falling by droplets but here the origin of the process is a competition between gravity and yield stress, which leads to larger "droplets". In order to clarify the representation of data we only plotted one symbol per level reached after each new droplet drop except when the duration between two successive drops was too long (in that case we plotted several points, which generally corresponds to an apparent stoppage of the flow).

We can see that despite a slightly higher yield stress, the flow of Carbopol through a given sieve is faster and stops before the flow of the emulsion through the same sieve. This means that the Carbopol solution needs a higher pressure drop to flow than the emulsion. Actually this effect is almost negligible for large mesh sizes (say, beyond $250 \mu \mathrm{m}$ (see Figure 13)) and increases when the mesh size decreases, becoming dramatic for a mesh size below $20 \mu \mathrm{m}$. In the latter case there is almost no flow observed through the sieve for the Carbopol while the flow is still significant for the emulsion.

These experiments show that even for a simpler geometry there is a difference between the flow characteristics observed for the two fluids as soon as the pore size becomes sufficiently small. It is very likely that this effect be at the origin of the differences observed between both fluids since, roughly speaking, the critical mesh size can be considered similar to that of the smallest pores. Thus there might be some kind of jamming of Carbopol blobs in the smallest pores, an effect which would occur for smaller mesh sizes for the emulsion made of smaller elements. 
However the point which remains very surprising is that the ratio between the values for $\alpha$ for the two fluids apparently remains constant over a wide range of pore size (related to bead diameter). If this was solely an effect of jamming it would be significant for small pores and would become negligible for sufficiently large pores. This means that further studies are needed to completely understand the origin of this effect.

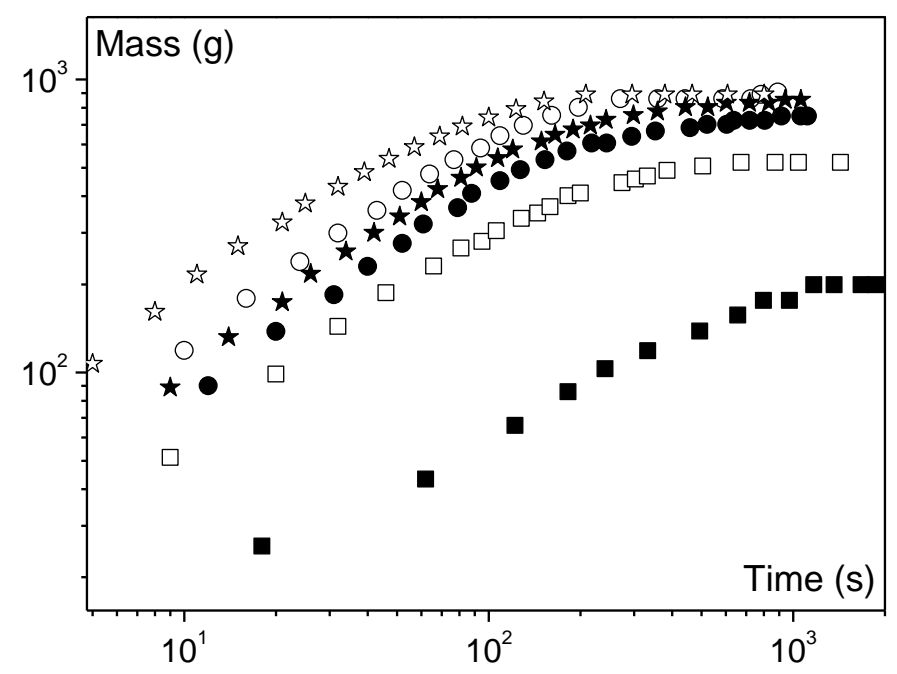

Figure 13: Mass evolution as a function of time for the flow of Carbopol solution ( $\tau_{c}=65 \mathrm{~Pa}$ ) (open symbols) and emulsion ( $\tau_{c}=76 \mathrm{~Pa}$ ) (filled symbols) through sieves of 20 (squares), 80 (circles), and $250 \mu m$ (stars).

\section{Conclusion}

We have carried out flow tests through well-calibrated porous media (glass beads), in which both the rheological behavior of the fluids and the flow characteristics through the porous medium (beads packing) were well-controlled. A thorough analysis of the data made it possible to extract the general pressure drop vs flow rate curve for a yield stress fluid flowing through a porous medium. This general law contains a yielding term which may be simply expressed as a function of the yield stress of the material and the bead size. The results obtained with the emulsion seem more reliable than those obtained with Carbopol solutions, which were likely to be affected by some jamming effects in the smallest pores. As for a Herschel-Bulkley model, an additional viscous term, depending on the flow rate, had to be added to the yielding term in order to obtain the total pressure drop during flow, which gives the general Darcy's law for yield stress fluid flowing through a bead packing:

$D \nabla p=5.5 \tau_{c}+85 k\left(\frac{V}{D}\right)^{n}$

This expression is expected to be valid only if the elements composing the yield stress fluid are much smaller than the pore size, which obviously can never be perfectly true. 
The yielding term in (14) is related to the mobilization of the fluid in the widest path throughout the porous medium. As the flow rate increases it is expected that additional thinner fluid paths progressively take part in the flow. This is proved by the fact that we can get an expression similar to (14) in the case of a series of parallel conduits of different sizes with a maximum diameter equal to $D$.

The above expression can be extrapolated to any kind of porous medium, under the condition that one is able to identify (i) the appropriate value for the maximum width of the widest path, which will govern the value of the coefficient $\alpha$, and (ii) the type of pore size distribution and structure, which will govern the value of $\beta$.

\section{References}

[1] R.P. Chhabra, J. Comiti, I. Machac, Flow of non-Newtonian fluids in fixed and fluidised beds, Chem. Eng. Sci. 56 (2001) 1-27.

[2] S. Liu, J. Masliyah, On non-Newtonian fluid flow in ducts and porous media, Chem. Eng. Sci. 53, (1998) 1175-1201.

[3] X. Lopez, P.H. Valvatne, M.J. Blunt, Predictive network modelling of single-phase nonNewtonian flow in porous media, J. Colloid Interface Sci. 264 (2003) 256-265.

[4] N.E. Sabiri, J. Comiti, Pressure drop in non-Newtonian purely viscous fluid flow through porous media, Chem. Eng. Sci. 50 (1995) 1193-1201.

[5] M. Chen, W. Rossen, Y.C. Yortsos, The flow and displacement in porous media of fluids with yield stress, Chem. Eng. Sci. 60 (2005) 4183-4202.

[6] P.L.J. Zitha, C.W. Botermans, J.v.d. Hoek, F.J. Vermolen, Control of flow through porous media using polymer gels, J. Appl. Phys. 92 (2002) 1143-1153.

[7] A.F. Morais, H. Seybold, H.J. Hermann, J.S. Andrade Jr., Non-Newtonian fluid flow through three-dimensional disordered porous media, Phys. Rev. Lett. 103 (2009) 194502.

[8] T. Al-Fariss and K.L. Pinder, Flow through porous media of a shear-thinning liquid with yield stress, Can. J. Chem. Eng. 65 (1987) 391-405.

[9] G.G. Chase, P. Dachavijit, A correlation for yield stress fluid flow through packed beds, Rheol. Acta 44 (2005) 495-501.

[10] H.C. Park, The flow of non-Newtonian fluids through porous media, Ph.D. thesis, Michigan State University (1972)

[11] H. Pascal, Nonsteady flow of non-Newtonian fluids through a porous medium, Int. J. Eng. Sci. 21 (3) (1983) 199-210.

[12] G.C. Vradis, A.L. Protopapas, Macroscopic conductivities for flow of Bingham plastics in porous media, J. Hydraul. Eng. 119 (1993) 95-108.

[13] M.T. Balhoff, and K.E. Thompson, Modeling the steady flow of yield stress fluids in packed beds, AIChE J. 50 (2004) 3034-3048.

[14] M. Sahimi, Non-linear transport processes in disordered media, AIChE J. 39 (1993) 369-386.

[15] T. Sochi, M.J. Blunt, Pore-scale network modelling of Ellis and Herschel-Bulkley fluids, J. Petr. Sci. Eng. 60 (2008) 105-124.

[16] P. Coussot, Rheometry of pastes, suspensions and granular materials (Wiley, New York, 2005) 
[17] J.M. Piau, Carbopol gels: elastoviscoplastic and slippery glasses made of individual swollen sponges meso- and macroscopic properties, constitutive equations and scaling laws, J. Non-Newt. Fluid Mech 144 (2007) 1-29.

[18] P. Coussot, H. Tabuteau, X. Chateau, L. Tocquer, G. Ovarlez, Aging and solid or liquid behavior in pastes, Journal of Rheology 50 (6) (2006) 975-994.

[19] T.G. Mason, J. Bibette, D.A. Weitz, Yielding and Flow of Monodisperse Emulsions, J. Colloid Interface Sci. 179 (1996) 439-448.

[20] P. Coussot , L. Tocquer, C. Lanos, G. Ovarlez, Macroscopic vs. local rheology of yield stress fluids, J. Non-Newt. Fluid Mech 158 (2009) 85-90.

[21] J.C. Dupla, J. Canou, D. Gouvenot, An advanced experimental set-up for studying monodirectional grout injection process, Proceedings of the ICE - Ground Improvement, 7 (2004), $1-9$.

[22] Z. Saada, J. Canou, L. Dormieux, J.C. Dupla, Evaluation of elementary filtration properties of a cement grout injected in a sand, Canadian Geotechnical Journal, 43, (2006) 1273-1289.

[23] Z. Saada, J. Canou, L. Dormieux, J.C. Dupla, S. Maghous, Modelling of cement suspension flow in granular porous media. International Journal for Numerical and Analytical Methods in Geomechanics, 29, (2005) 691-711.

[24] J. Kozeny, Über Kapillare Leitung des Wassers im Boden, Sitzungsber, Akad. Wiss. Wien 136 (1927) 271-306.

[25] R.B. Bird, G. Dai, B. J. Yarusso, The rheology and flow of viscoplastic materials, Rev. Chemical Eng. 1 (1982) 1-70. 